\title{
A note on complete hyperbolic operators with log- Zygmund coefficients
}

\author{
Ferruccio Colombini, Daniele Del Santo, Francesco Fanelli and Guy \\ Métivier
}

\begin{abstract}
The present paper is the continuation of the recent work [7], and it is devoted to strictly hyperbolic operators with non-regular coefficients. We focus here on the case of complete operators whose second order coefficients are log-Zygmund continuous in time, and we investigate the $H^{\infty}$ well-posedness of the associate Cauchy problem.
\end{abstract}

Mathematics Subject Classification (2000). Primary 35L15; Secondary 35B65, 35S50, 35B45.

Keywords. Hyperbolic operators, non-Lipschitz coefficient, log-Zygmund regularity, energy estimates, well-posedness.

\section{Introduction}

The present paper is, in a certain sense, the continuation of the recent work [7]: it is devoted to the study of strictly hyperbolic operators with log-Zygmund in time coefficients. In particular, we want to prove the $H^{\infty}$ well-posedness of the Cauchy problem related to a complete second order operator,

$$
L u=\partial_{t}^{2} u-\sum_{i, j=1}^{N} \partial_{i}\left(a_{i j}(t, x) \partial_{j} u\right)+\sum_{j=0}^{N} b_{j}(t, x) \partial_{j} u+c(t, x) u,
$$

(with $\partial_{0}=\partial_{t}$ ) whose highest order coefficients satisfy such a regularity assumption (see also relation (2.3) below).

The core of the proof is to establish suitable energy estimates for $L$.

It is well-known (see [14]; see also e.g. [13, Ch. IX] or [18, Ch. 6] for analogous results) that, if the coefficients $a_{j k}$ are Lipschitz continuous with respect to $t$ and only measurable in $x$, then the Cauchy problem for $L$ is well-posed in $H^{1} \times L^{2}$. If the $a_{j k}$ 's are Lipschitz continuous with respect to $t$ and $\mathcal{C}_{b}^{\infty}$ (i.e. $\mathcal{C}^{\infty}$ and bounded with all their derivatives) with respect to the space variables, one can recover the 
well-posedness in $H^{s+1} \times H^{s}$ for all $s \in \mathbb{R}$. Moreover, in the latter case, one gets, for all $s \in \mathbb{R}$ and for a constant $C_{s}$ depending only on it, the following energy estimate:

$$
\begin{aligned}
& \sup _{0 \leq t \leq T}\left(\|u(t, \cdot)\|_{H^{s+1}}+\left\|\partial_{t} u(t, \cdot)\right\|_{H^{s}}\right) \leq \\
& \leq C_{s}\left(\|u(0, \cdot)\|_{H^{s+1}}+\left\|\partial_{t} u(0, \cdot)\right\|_{H^{s}}+\int_{0}^{T}\|L u(t, \cdot)\|_{H^{s}} d t\right)
\end{aligned}
$$

for all $u \in \mathcal{C}\left([0, T] ; H^{s+1}\left(\mathbb{R}^{N}\right)\right) \cap \mathcal{C}^{1}\left([0, T] ; H^{s}\left(\mathbb{R}^{N}\right)\right)$ for which $L u$ belongs to $L^{1}\left([0, T] ; H^{s}\left(\mathbb{R}^{N}\right)\right)$. Let us explicitly remark that previous inequality involves no loss of regularity for the function $u$ : estimate (1.2) holds for every $u$ of class $\mathcal{C}^{2}\left([0, T] ; H^{\infty}\left(\mathbb{R}^{N}\right)\right)$ and the Cauchy problem for $L$ is well-posed in $H^{\infty}$ with no loss of derivatives.

If the Lipschitz continuity (in time) hypothesis is not fulfilled, then (1.2) is no more true. Nevertheless, one can still try to recover $H^{\infty}$-well-posedness, with a loss of derivatives in the energy estimate.

The first case to consider is the case of the coefficients $a_{j k}$ depending only on $t$ :

$$
L u=\partial_{t}^{2} u-\sum_{j, k=1}^{N} a_{j k}(t) \partial_{j} \partial_{k} u .
$$

In [5], Colombini, De Giorgi and Spagnolo assumed the coefficients to satisfy an integral log-Lipschitz condition:

$$
\int_{0}^{T-\varepsilon}\left|a_{j k}(t+\varepsilon)-a_{j k}(t)\right| d t \leq C \varepsilon \log \left(1+\frac{1}{\varepsilon}\right),
$$

for some constant $C>0$ and all $\varepsilon \in] 0, T]$. They obtained an energy estimate with a fixed loss of derivatives: there exists a constant $\delta>0$ such that, for all $s \in \mathbb{R}$, the inequality

$$
\begin{aligned}
& \sup _{0 \leq t \leq T}\left(\|u(t, \cdot)\|_{H^{s+1-\delta}}+\left\|\partial_{t} u(t, \cdot)\right\|_{H^{s-\delta}}\right) \leq \\
& \leq C_{s}\left(\|u(0, \cdot)\|_{H^{s+1}}+\left\|\partial_{t} u(0, \cdot)\right\|_{H^{s}}+\int_{0}^{T}\|L u(t, \cdot)\|_{H^{s}} d t\right)
\end{aligned}
$$

holds true for all $u \in \mathcal{C}^{2}\left([0, T] ; H^{\infty}\left(\mathbb{R}^{N}\right)\right)$, for some constant $C_{s}$ depending only on $s$.

Let us explain briefly the main ideas of the proof, which are actually the basis to establish also the next results we are going to quote. The authors first smoothed out the coefficients using a mollifier kernel $\left(\rho_{\varepsilon}\right)_{\varepsilon}$. Then, by Fourier transform, they defined an approximated energy $E_{\varepsilon}(\xi, t)$ in the phase space, where the problem becomes a family of ordinary differential equations. At that point, the key step was to perform a different approximation of the coefficients in different zones of the 
phase space, which was realized setting $\varepsilon=|\xi|^{-1}$. This particular choice allowed them to obtain the result.

Let us remark that if the coefficients $a_{j k}$ are not Lipschitz continuous then a loss of regularity cannot be avoided. In fact, in paper [3], Cicognani and Colombini proved that, if the regularity of the coefficients $a_{j k}$ is measured by a modulus of continuity, any intermediate modulus of continuity between the Lipschitz and the $\log$-Lipschitz ones entails necessarily a loss of regularity, which, however, can be made arbitrarily small.

Recently Tarama (see paper [19]) was able to prove an analogous result, and the consequent $H^{\infty}$ well-posedness of the related Cauchy problem, when the coefficients satisfy an integral log-Zygmund condition: there exists a constant $C>$ 0 such that, for all $j, k$ and all $\varepsilon \in] 0, T / 2[$, one has

$$
\int_{\varepsilon}^{T-\varepsilon}\left|a_{j k}(t+\varepsilon)+a_{j k}(t-\varepsilon)-2 a_{j k}(t)\right| d t \leq C \varepsilon \log \left(1+\frac{1}{\varepsilon}\right) .
$$

It's obvious that, if the $a_{j k}$ 's satisfy (1.3), then they satisfy also (1.5): so, a more general class of functions is considered. The improvement with respect to paper [5], however, was obtained defining a new energy, which involved (by derivation in time) second derivatives of the approximated coefficients.

In paper [10], Colombini and Lerner considered instead the case when the coefficients $a_{j k}$ depend both on time and space variables. In particular, they assumed an isotropic punctual log-Lipschitz condition, i.e. there exists a constant $C>0$ such that, for all $\zeta=(\tau, \xi) \in \mathbb{R} \times \mathbb{R}^{N}, \zeta \neq 0$, one has

$$
\sup _{z=(t, x) \in \mathbb{R}^{\prime} \times \mathbb{R}^{N}}\left|a_{j k}(z+\zeta)-a_{j k}(z)\right| \leq C|\zeta| \log \left(1+\frac{1}{|\zeta|}\right) .
$$

The analysis was based on a broad use of the Littlewood-Paley decomposition and paradifferential calculus. As a matter of facts, one cannot use the Fourier transform, due to the dependence of $a_{j k}$ on $x$. Also in this case, a different approximation of the coefficients in different zones of the phase space was required: this was obtained setting $\varepsilon=2^{-\nu}$, where $2^{\nu}$ is the "size" of the frequencies in the $\nu$-th ring of the Littlewood-Paley decomposition (see also section 3). In the end, they got the following statement: for all $s \in] 0,1 / 4]$, there exist positive constants $\beta$ and $C_{s}$ and a time $\left.\left.T^{*} \in\right] 0, T\right]$ such that

$$
\begin{aligned}
& \sup _{0 \leq t \leq T^{*}}\left(\|u(t, \cdot)\|_{H^{-s+1-\beta t}}+\left\|\partial_{t} u(t, \cdot)\right\|_{H^{-s-\beta t}}\right) \leq \\
& \leq C_{s}\left(\|u(0, \cdot)\|_{H^{-s+1}}+\left\|\partial_{t} u(0, \cdot)\right\|_{H^{-s}}+\int_{0}^{T^{*}}\|L u(t, \cdot)\|_{H^{-s-\beta t}} d t\right)
\end{aligned}
$$

for all $u \in \mathcal{C}^{2}\left([0, T] ; H^{\infty}\left(\mathbb{R}^{N}\right)\right)$. Let us point out that the bound on $s$ was due to this reason: the product by a log-Lipschitz function is well-defined in $H^{s}$ if and only if $|s|<1$. Note also that this fact gives us a bound on the lifespan of the solution: the regularity index $-s+1-\beta T^{*}$ has to be strictly positive, so one 
can expect only local in time existence of a solution. Moreover in the case the coefficients $a_{j k}$ are $\mathcal{C}_{b}^{\infty}$ in space, the authors proved inequality (1.6) for all $s$ : so, they got well-posedness in $H^{\infty}$ with a loss of derivatives increasing in time.

The case of a complete strictly hyperbolic second order operator,

$$
L u=\sum_{j, k=0}^{N} \partial_{y_{j}}\left(a_{j k} \partial_{y_{k}} u\right)+\sum_{j=0}^{N}\left(b_{j} \partial_{y_{j}} u+\partial_{y_{j}}\left(c_{j} u\right)\right)+d u
$$

(here we set $y=(t, x) \in \mathbb{R}_{t} \times \mathbb{R}_{x}^{N}$ ), was considered by Colombini and Métivier in [11]. They assumed the same isotropic log-Lipschitz condition of [10] on the coefficients of the second order part of $L$, while $b_{j}$ and $c_{j}$ were supposed to be $\alpha$ Hölder continuous (for some $\alpha \in] 1 / 2,1[$ ) and $d$ to be only bounded. The authors headed towards questions such as local-in-space existence and uniqueness, and finite propagation speed.

Trying to mix up up a Tarama-like hypothesis (concerning the dependence on the time variable) with the one of Colombini and Lerner (with respect to $x$ ) is at this point a natural step. More precisely, we want to consider a pointwise log-Zygmund condition in time and a pointwise log-Lipschitz condition in space, uniformly with respect to the other variable (see conditions (2.3) and (2.4) below). Under this assumption, it's possible to prove an energy estimate analogous to (1.6). The first result in this direction was due to Colombini and Del Santo, in paper [6] (see also [12], where smoothness in space was however required). Nevertheless, they had to restrict themselves to the case of space dimension $N=1$ (see also [9] for the case of a complete operator). The result in the general instance $N \geq 1$ was only recently proved in paper [7]. Even if the difficulty of the additional space variables seems to be just technical, the approach assumed in [7], i.e. of paradifferential calculus depending on parameters, actually gives a more general and complete point of view of the problem.

The aim of the present note is to extend the result of [7] for homogeneous second order operators to the case where also lower order terms come into play. In particular, according with the results of [10], [11] and [9], we will assume the first order coefficients to be Hölder continuous in the space variable, and the zero order coefficient to be just bounded. From an energy estimate analogous to (1.6), we will infer, for smooth in space coefficients, the $H^{\infty}$ well-posedness of the related Cauchy problem with a finite loss of derivatives.

To complete the general picture of the state of the art, let us recall that in the already quoted paper [19], Tarama proved also an energy estimate with no loss of derivatives for coefficients $a_{j k}(t)$ which fulfill a (integral) Zygmund hypothesis. This result has been recently generalized in [8] to the case of $a_{j k}$ 's Zygmund continuous both in $t$ and $x$ (assuming also Hölder first order coefficients and bounded zero order coefficient), but only in the space $H^{1 / 2} \times H^{-1 / 2}$. If an isotropic Zygmund assumption implies or not loss of regularity for general initial data in $H^{s} \times H^{s-1}$ is still an open problem.

Our paper is organized in the following way. 
In the next section, we will state our main results.

A brief presentation of the tools we need in order to prove them will follow, in Section 3: we refer to [7] for a complete description. These tools mostly come from Fourier analysis. In particular, we will recall some basic facts on LittlewoodPaley decomposition and paradifferential calculus, both in its classical version (see e.g. [1], [2] and [16]) and in the one depending on a parameter (see [15] and [17]). We will also recall some basic facts on functions with low regularity modulus of continuity.

Section 4 is devoted to the proof of energy estimates for our strictly hyperbolic operator with non regular (both in $t$ and $x$ ) coefficients: this is the most involving part. We will just give a sketch of the analysis of the higher order terms (already treated in [7]), while we will give the most of the details about the lower order ones.

Finally, in Section 5 we will show how the just proved energy estimates allow to recover the $H^{\infty}$ well-posedness of the related Cauchy problem, in the case of coefficients which are smooth in the space variables.

\section{Main results}

Let us consider the operator over $\left[0, T_{0}\right] \times \mathbb{R}^{N}$ (for some $T_{0}>0$ and $N \geq 1$ )

$$
L u=\partial_{t}^{2} u-\sum_{i, j=1}^{N} \partial_{i}\left(a_{i j}(t, x) \partial_{j} u\right)+\sum_{j=0}^{N} b_{j}(t, x) \partial_{j} u+c(t, x) u
$$

(with the notation $\partial_{0}=\partial_{t}$ ) and let us suppose $L$ to be strictly hyperbolic with bounded coefficients, i.e. there exist two positive constants $0<\lambda_{0} \leq \Lambda_{0}$ such that, for all $(t, x) \in \mathbb{R}_{t} \times \mathbb{R}_{x}^{N}$ and all $\xi \in \mathbb{R}^{N}$, one has

$$
\lambda_{0}|\xi|^{2} \leq \sum_{i, j=1}^{N} a_{i j}(t, x) \xi_{i} \xi_{j} \leq \Lambda_{0}|\xi|^{2} .
$$

Moreover, assume that the $a_{i j}$ 's are log-Zygmund continuous in the time variable, uniformly with respect to $x$ : there exists a constant $K_{0}>0$ such that, for all $\tau>0$, one has

$$
\sup _{(t, x)}\left|a_{i j}(t+\tau, x)+a_{i j}(t-\tau, x)-2 a_{i j}(t, x)\right| \leq K_{0} \tau \log \left(1+\frac{1}{\tau}\right) .
$$
result.

The goal of the present paper is to prove the following $H^{\infty}$ well-posedness

Theorem 2.1. Consider the operator $L$ defined in (2.1), under the strictly hyperbolicity condition (2.2).

Let us suppose the following conditions on the coefficients of $L$ :

(i) the $a_{i j}$ 's are log-Zygmund continuous in time, i.e. (2.3) holds;

(ii) the $a_{i j}$ 's are also of class $\mathcal{C}_{b}^{\infty}$ with respect to $x$; 
(iii) $b_{j}$, for all $0 \leq j \leq N$, and $c$ belong to $L^{\infty}\left(\left[0, T_{0}\right] ; \mathcal{C}_{b}^{\infty}\left(\mathbb{R}^{N}\right)\right)$.

Then the Cauchy problem for $L$,

$$
\left\{\begin{array}{l}
L u=f \\
u_{\mid t=0}=u_{0}, \quad \partial_{t} u_{\mid t=0}=u_{1},
\end{array}\right.
$$

is well-posed in $H^{\infty}\left(\mathbb{R}^{N}\right)$, globally in time.

Recall that the notation $f \in \mathcal{C}_{b}^{\infty}$ means that $f$ is of class $\mathcal{C}^{\infty}$, and it is bounded with all its derivatives.

The proof of the previous result is essentially based on finding energy estimates for $L$ : we will establish them when the coefficients satisfy minimal regularity assumptions also with respect to the space variable. This means that we will focus on log-Lipschitz continuous $a_{i j}$ 's and Hölder continuous $b_{j}$ 's, while the 0 -th order coefficient $c$ can be taken just bounded (see also [10], [11] and [9] about these hypothesis).

So, assume that there exists a constant $K_{1}>0$ such that

$$
\sup _{(t, x)}\left|a_{i j}(t, x+y)-a_{i j}(t, x)\right| \leq K_{1}|y| \log \left(1+\frac{1}{|y|}\right)
$$

for all $y \in \mathbb{R}^{N} \backslash\{0\}$. Suppose also the following on the lower order terms:

$$
b_{j} \in L^{\infty}\left(\left[0, T_{0}\right] ; \mathcal{C}^{\omega}\left(\mathbb{R}^{N}\right)\right), 0 \leq j \leq N, \quad \text { and } \quad c \in L^{\infty}\left(\left[0, T_{0}\right] \times \mathbb{R}^{N}\right),
$$

for some real number $0<\omega \leq 1$ (with $\mathcal{C}_{*}^{\omega}:=B_{\infty, \infty}^{\omega}$ if $\omega=1$ ).

Theorem 2.2. Let $L$ be the operator defined in (2.1), whose coefficients fulfill conditions (2.2), (2.3), (2.4) and (2.5).

Then, for all fixed $\theta \in] 0, \min \{\omega, 1\}\left[\right.$, there exist a constant $\beta^{*}>0$, a time $T>0$ and a constant $C>0$ such that the estimate

$$
\begin{aligned}
& \sup _{0 \leq t \leq T}\left(\|u(t, \cdot)\|_{H^{-\theta+1-\beta^{*} t}}+\left\|\partial_{t} u(t, \cdot)\right\|_{H^{-\theta-\beta^{*} t}}\right) \leq \\
& \leq C\left(\|u(0, \cdot)\|_{H^{-\theta+1}}+\left\|\partial_{t} u(0, \cdot)\right\|_{H^{-\theta}}+\int_{0}^{T}\|L u(t, \cdot)\|_{H^{-\theta-\beta^{*}}} d t\right)
\end{aligned}
$$

holds true for all $u \in \mathcal{C}^{2}\left([0, T] ; H^{\infty}\left(\mathbb{R}^{N}\right)\right)$.

We will exlpain in Section 5 how one can recover the $H^{\infty}$ well-posedness for $(C P)$ from the energy estimates of Theorem 2.2.

\section{Functional toolbox}

The present section aims to give an overview of the tools, based on Fourier analysis techniques, we need to prove our results. We refer to paper [7] (in particular Section 3 ) and the references therein for a more complete and detailed treatment. 
Let us start with a definition. We $\operatorname{set}^{1} \Pi(D):=\log (2+|D|)$, i.e. its symbol is $\pi(\xi):=\log (2+|\xi|)$.

Definition 3.1. For all $\alpha \in \mathbb{R}$, we define the space $H^{s+\alpha} \log$ as $\Pi^{-\alpha} H^{s}$, i.e.

$$
f \in H^{s+\alpha \log } \Leftrightarrow \Pi^{\alpha} f \in H^{s} \quad \Leftrightarrow \quad \pi^{\alpha}(\xi)\left(1+|\xi|^{2}\right)^{s / 2} \widehat{f}(\xi) \in L^{2} .
$$

These logarithmic Sobolev spaces were introduced in [11]. Due to the low regularity of the coefficients of $L$, this kind of spaces will come into play in our computations.

\subsection{Littlewood-Paley theory and paradifferential calculus}

Let us now define the so called Littlewood-Paley decomposition, based on a nonhomogeneous dyadic partition of unity with respect to the Fourier variable. We refer to [1], [2] and [16] for the details.

So, fix a smooth radial function $\chi$ supported in the ball $B(0,2)$, equal to 1 in a neighborhood of $B(0,1)$ and such that $r \mapsto \chi(r e)$ is nonincreasing over $\mathbb{R}_{+}$ for all unitary vectors $e \in \mathbb{R}^{N}$. Set also $\varphi(\xi)=\chi(\xi)-\chi(2 \xi)$.

The dyadic blocks $\left(\Delta_{j}\right)_{j \in \mathbb{Z}}$ are defined by

$$
\Delta_{j}:=0 \text { if } j \leq-1, \quad \Delta_{0}:=\chi(D) \text { and } \Delta_{j}:=\varphi\left(2^{-j} D\right) \text { if } j \geq 1 .
$$

We also introduce the following low frequency cut-off operator:

$$
S_{j} u:=\chi\left(2^{-j} D\right)=\sum_{k \leq j} \Delta_{k} \quad \text { for } \quad j \geq 0 .
$$

The following classical properties will be used freely throughout the paper:

- for any $u \in \mathcal{S}^{\prime}$, the equality $u=\sum_{j} \Delta_{j} u$ holds true in $\mathcal{S}^{\prime}$;

- for all $u$ and $v$ in $\mathcal{S}^{\prime}$, the sequence $\left(S_{j-3} u \Delta_{j} v\right)_{j \in \mathbb{N}}$ is spectrally supported in dyadic annuli.

Let us also mention a fundamental result, which explains, by the so-called Bernstein's inequalities, the way in which derivatives act on spectrally localized functions.

Lemma 3.2. Let $0<r<R$. A constant $C$ exists so that, for any nonnegative integer $k$, any couple $(p, q)$ in $[1,+\infty]^{2}$ with $p \leq q$ and any function $u \in L^{p}$, we have, for all $\lambda>0$,

(i) $\operatorname{supp} \widehat{u} \subset B(0, \lambda R) \quad \Longrightarrow \quad\left\|\nabla^{k} u\right\|_{L^{q}} \leq C^{k+1} \lambda^{k+N\left(\frac{1}{p}-\frac{1}{q}\right)}\|u\|_{L^{p}} ;$

(ii) $\operatorname{supp} \widehat{u} \subset\left\{\xi \in \mathbb{R}^{N}|r \lambda \leq| \xi \mid \leq R \lambda\right\}$

$$
\Longrightarrow \quad C^{-k-1} \lambda^{k}\|u\|_{L^{p}} \leq\left\|\nabla^{k} u\right\|_{L^{p}} \leq C^{k+1} \lambda^{k}\|u\|_{L^{p}}
$$

By the Littlewood-Paley decomposition, we can characterize the new Sobolev class introduced in Definition 3.1 (see [16, Prop. 4.1.11]).

Proposition 3.3. Let $s, \alpha \in \mathbb{R}$. A tempered distribution $u$ belongs to the space $H^{s+\alpha} \log$ if and only if:

\footnotetext{
${ }^{1}$ Throughout we agree that $f(D)$ stands for the pseudo-differential operator $u \mapsto \mathcal{F}^{-1}(f \mathcal{F} u)$.
} 
(i) for all $k \in \mathbb{N}, \Delta_{k} u \in L^{2}\left(\mathbb{R}^{N}\right)$;

(ii) setting $\delta_{k}:=2^{k s}(1+k)^{\alpha}\left\|\Delta_{k} u\right\|_{L^{2}}$ for all $k \in \mathbb{N}$, then $\left(\delta_{k}\right)_{k} \in \ell^{2}(\mathbb{N})$.

Moreover, $\|u\|_{H^{s+\alpha} \log }$ is equivalent to $\left\|\left(\delta_{k}\right)_{k}\right\|_{\ell^{2}}$.

Littlewood-Paley decomposition allows also to define operators associated to low regularity symbols, via the so-called paradifferential calculus. For reasons which will appear clear in the sequel, we have to resort not to the classical version, as introduced by Bony in [2], but to the one depending on a parameter $\gamma \geq 1$ (see e.g. [17] and [15]). Let us recall here the main ideas of the construction.

Hence, fix $\gamma \geq 1$ and take a cut-off function $\psi_{\gamma} \in \mathcal{C}^{\infty}\left(\mathbb{R}^{N} \times \mathbb{R}^{N}\right)$ which verifies the following properties:

- there exist $0<\varepsilon_{1}<\varepsilon_{2}<1$ such that

$$
\psi_{\gamma}(\eta, \xi)=\left\{\begin{array}{lll}
1 & \text { for } & |\eta| \leq \varepsilon_{1}(\gamma+|\xi|) \\
0 & \text { for } \quad|\eta| \geq \varepsilon_{2}(\gamma+|\xi|)
\end{array}\right.
$$

- for all $(\beta, \alpha) \in \mathbb{N}^{N} \times \mathbb{N}^{N}$, there exists a constant $C_{\beta, \alpha}$ such that

$$
\left|\partial_{\eta}^{\beta} \partial_{\xi}^{\alpha} \psi_{\gamma}(\eta, \xi)\right| \leq C_{\beta, \alpha}(\gamma+|\xi|)^{-|\alpha|-|\beta|} .
$$

Remark 3.4. We remark that $\varepsilon_{1}, \varepsilon_{2}$ and the different $C_{\beta, \alpha}$ 's occurring in the previous estimates must not depend on $\gamma$.

For instance, if $\gamma=1$, one can take

$$
\psi_{\gamma}(\eta, \xi) \equiv \psi_{1}(\eta, \xi):=\sum_{k=0}^{+\infty} \chi_{k-3}(\eta) \varphi_{k}(\xi)
$$

where $\chi$ and $\varphi$ are the localization (in the phase space) functions associated to a Littlewood-Paley decomposition. Similarly, if $\gamma>1$ it is possible to find a suitable integer $\mu \geq 0$ such that

$$
\psi_{\gamma}(\eta, \xi):=\chi_{\mu}(\eta) \chi_{\mu+2}(\xi)+\sum_{k=\mu+3}^{+\infty} \chi_{k-3}(\eta) \varphi_{k}(\xi)
$$

is a function with the properties just described.

Define now $G^{\psi_{\gamma}}$ as the inverse Fourier transform of $\psi_{\gamma}$ with respect to the variable $\eta$ :

$$
G^{\psi_{\gamma}}(x, \xi):=\left(\mathcal{F}_{\eta}^{-1} \psi_{\gamma}\right)(x, \xi) .
$$

Lemma 3.5. For all $(\beta, \alpha) \in \mathbb{N}^{N} \times \mathbb{N}^{N}$, there exist constants $C_{\beta, \alpha}$, independent of $\gamma$, such that:

$$
\begin{aligned}
&\left\|\partial_{x}^{\beta} \partial_{\xi}^{\alpha} G^{\psi_{\gamma}}(\cdot, \xi)\right\|_{L^{1}\left(\mathbb{R}_{x}^{N}\right)} \leq C_{\beta, \alpha}(\gamma+|\xi|)^{-|\alpha|+|\beta|} \\
&\left\||\cdot| \log \left(2+\frac{1}{|\cdot|}\right) \partial_{x}^{\beta} \partial_{\xi}^{\alpha} G^{\psi_{\gamma}}(\cdot, \xi)\right\|_{L^{1}\left(\mathbb{R}_{x}^{N}\right)} \leq C_{\beta, \alpha}(\gamma+|\xi|)^{-|\alpha|+|\beta|-1} \cdot(3.3) \\
& \cdot \log (1+\gamma+|\xi|) .
\end{aligned}
$$


Thanks to $G^{\psi_{\gamma}}$, we can smooth out a symbol $a$ in the $x$ variable and then define the paradifferential operator associated to $a$ as the pseudodifferential operator related to this smooth function. We set the classical symbol associated to $a$ to be

$$
\sigma_{a}(x, \xi):=\left(\psi_{\gamma}\left(D_{x}, \xi\right) a\right)(x, \xi)=\left(G^{\psi_{\gamma}}(\cdot, \xi) *_{x} a(\cdot, \xi)\right)(x),
$$

and then the paradifferential operator associated to $a$ :

$$
T_{a}:=\sigma_{a}\left(x, D_{x}\right),
$$

where we have omitted $\psi_{\gamma}$ because the definition is independent of it, up to lower order terms (see [17]).

Remark 3.6. Let us note that if $a=a(x) \in L^{\infty}$ and if we take the cut-off function $\psi_{1}$, then $T_{a}$ is actually the usual paraproduct operator. If we take $\psi_{\gamma}$ as defined in (3.1), instead, we get a paraproduct operator which starts from high enough frequencies, which will be indicated with $T_{a}^{\mu}$ (see [11, Par. 3.3]).

Let us point out that we can also define a $\gamma$-dyadic decomposition. First of all, we set

$$
\Lambda(\xi, \gamma):=\left(\gamma^{2}+|\xi|^{2}\right)^{1 / 2}
$$

Then, taken the usual smooth function $\chi$ associated to a Littlewood-Paley decomposition, we define

$$
\chi_{\nu}(\xi, \gamma):=\chi\left(2^{-\nu} \Lambda(\xi, \gamma)\right), \quad S_{\nu}^{\gamma}:=\chi_{\nu}\left(D_{x}, \gamma\right), \quad \Delta_{\nu}^{\gamma}:=S_{\nu+1}^{\gamma}-S_{\nu}^{\gamma} .
$$

The usual properties of the support of the localization functions still hold, and for all fixed $\gamma \geq 1$ and all tempered distributions $u$, we have

$$
u=\sum_{\nu=0}^{+\infty} \Delta_{\nu}^{\gamma} u \quad \text { in } \quad \mathcal{S}^{\prime} .
$$

Moreover, with natural modifications in definitions, we can introduce the space $H_{\gamma}^{s+\alpha \log }$ as the set of tempered distributions for which

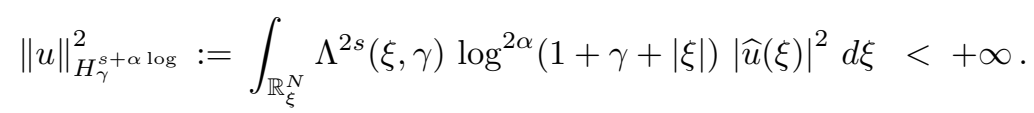

For the details see [17, Appendix B]. What is important to retain is that, once we fix $\gamma \geq 1$ (for example, to obtain positivity of paradifferential operators involved in our computations), all the previous construction is equivalent to the classical one; in particular, the space $H_{\gamma}^{s+\alpha \log }$ coincides with $H^{s+\alpha \log }$, the respective norms are equivalent and the characterization given by Proposition 3.3 still holds true.

\subsection{On log-Lipschitz and log-Zygmund functions}

Let us now recall some definitions and properties about the low regularity conditions we assume on the second order coefficients of $L$. If not otherwise specified, one can find all the proofs to next results in [7, Section 3]. 
Definition 3.7. A function $f \in L^{\infty}\left(\mathbb{R}^{N}\right)$ is said to be log-Lipschitz, and we write $f \in L L\left(\mathbb{R}^{N}\right)$, if the quantity

$$
|f|_{L L}:=\sup _{x, y \in \mathbb{R}^{N},|y|<1}\left(\frac{|f(x+y)-f(x)|}{|y| \log \left(1+\frac{1}{|y|}\right)}\right)<+\infty .
$$

We set $\|f\|_{L L}:=\|f\|_{L^{\infty}}+|f|_{L L}$.

Let us define also the space of log-Zygmund functions. We will give the general definition in $\mathbb{R}^{N}$, even if the one dimensional case will be the only relevant one for our purposes.

Definition 3.8. A function $g \in L^{\infty}\left(\mathbb{R}^{N}\right)$ is said to be log-Zygmund, and we write $g \in L Z\left(\mathbb{R}^{N}\right)$, if the quantity

$$
|g|_{L Z}:=\sup _{x, y \in \mathbb{R}^{N},|y|<1}\left(\frac{|g(x+y)+g(x-y)-2 g(x)|}{|y| \log \left(1+\frac{1}{|y|}\right)}\right)<+\infty .
$$

We set $\|g\|_{L Z}:=\|g\|_{L^{\infty}}+|g|_{L Z}$.

Remark 3.9. Let us immediately point out that, by monotonicity of logarithmic function, we can replace the factor $\log (1+1 /|y|)$ in previous definitions with $\log (1+\gamma+1 /|y|)$, for all parameters $\gamma \geq 1$. As paradifferential calculus with parameters will play a fundamental role in our computations, it's convenient to perform such a change, and so does also in hypothesis (2.3) and (2.4) of section 2 .

Let us recall also the following characterization of the space $L Z$.

Proposition 3.10. The space $L Z\left(\mathbb{R}^{N}\right)$ coincides with the logarithmic Besov space $B_{\infty, \infty}^{1-\log }$, i.e. the space of tempered distributions $u$ such that

$$
\sup _{\nu \geq 0}\left(2^{\nu}(\nu+1)^{-1}\left\|\Delta_{\nu} u\right\|_{L^{\infty}}\right)<+\infty .
$$

On the one hand, from definitions 3.7 and 3.8, it's obvious that $L L\left(\mathbb{R}^{N}\right) \hookrightarrow$ $L Z\left(\mathbb{R}^{N}\right)$ : Proposition 3.3 of [10] explains this property in terms of dyadic decomposition.

Proposition 3.11. There exists a constant $C$ such that, for all $a \in L L\left(\mathbb{R}^{N}\right)$ and all integers $k \geq 0$, we have

$$
\left\|\Delta_{k} a\right\|_{L^{\infty}} \leq C(k+1) 2^{-k}\|a\|_{L L} .
$$

Moreover, for all $k \in \mathbb{N}$ we have

$$
\begin{aligned}
\left\|a-S_{k} a\right\|_{L^{\infty}} & \leq C(k+1) 2^{-k}\|a\|_{L L} \\
\left\|S_{k} a\right\|_{\mathcal{C}^{0,1}} & \leq C(k+1)\|a\|_{L L} .
\end{aligned}
$$


Recall that, again from Proposition 3.3 of [10], property (3.7) is a characterization of the space $L L$.

On the other hand, we have also $B_{\infty, \infty}^{1} \hookrightarrow L L$ : the Zygmund condition entails a logarithmic loss in the first variation. As the next lemma states, this instance actually occurs also for log-Zygmund functions. For our purposes, it's enough to consider a log-Zygmund function $a$ depending only on the time variable $t$, but the same reasoning holds also in higher dimensions.

Lemma 3.12. For all $a \in L Z(\mathbb{R})$, there exists a constant $C$, depending only on the LZ norm of $a$, such that, for all $\gamma \geq 1$ and all $0<|\tau|<1$ one has

$$
\sup _{t \in \mathbb{R}}|a(t+\tau)-a(t)| \leq C|\tau| \log ^{2}\left(1+\gamma+\frac{1}{|\tau|}\right) .
$$

Now, given a log-Zygmund function $a(t)$, we can regularize it by convolution. So, take an even function $\rho \in \mathcal{C}_{0}^{\infty}\left(\mathbb{R}_{t}\right), 0 \leq \rho \leq 1$, whose support is contained in the interval $[-1,1]$ and such that $\int \rho(t) d t=1$, and define the mollifier kernel

$$
\left.\left.\rho_{\varepsilon}(t):=\frac{1}{\varepsilon} \rho\left(\frac{t}{\varepsilon}\right) \quad \forall \varepsilon \in\right] 0,1\right] .
$$

We smooth out the function $a$ setting, for all $\varepsilon \in] 0,1]$,

$$
a_{\varepsilon}(t):=\left(\rho_{\varepsilon} * a\right)(t)=\int_{\mathbb{R}_{s}} \rho_{\varepsilon}(t-s) a(s) d s .
$$

The following proposition holds.

Proposition 3.13. Let $a \in L Z(\mathbb{R})$. There exist constants $C$ such that, for all $\gamma \geq 1$,

$$
\begin{aligned}
\left|a_{\varepsilon}(t)-a(t)\right| & \leq C\|a\|_{L Z} \varepsilon \log \left(1+\gamma+\frac{1}{\varepsilon}\right) \\
\left|\partial_{t} a_{\varepsilon}(t)\right| & \leq C\|a\|_{L Z} \log ^{2}\left(1+\gamma+\frac{1}{\varepsilon}\right) \\
\left|\partial_{t}^{2} a_{\varepsilon}(t)\right| & \leq C\|a\|_{L Z} \frac{1}{\varepsilon} \log \left(1+\gamma+\frac{1}{\varepsilon}\right) .
\end{aligned}
$$

\subsection{Low regularity symbols and calculus}

Let us now consider symbols $a(t, x, \xi)$, which are $L Z_{t}-L L_{x}$, and investigate properties of the corresponding paradifferential operators, built as explained above.

As we will have to make use of the paradifferential calculus with parameters, we will allow the symbols to depend also on some $\gamma \geq 1$.

We point out that in our calculus the time can be treated as an additional parameter, while $\xi$ represents, as usual, the dual variable to $x$.

Definition 3.14. A symbol of order $m+\delta \log ($ for $m, \delta \in \mathbb{R}$ ) is a function $a(t, x, \xi, \gamma)$ which is locally bounded on $\left[0, T_{0}\right] \times \mathbb{R}^{N} \times \mathbb{R}^{N} \times\left[1,+\infty\left[\right.\right.$, of class $\mathcal{C}^{\infty}$ with respect to 
$\xi$ and which satisfy the following property: for all $\alpha \in \mathbb{N}^{N}$, there exists a constant $C_{\alpha}>0$ such that

$$
\left|\partial_{\xi}^{\alpha} a(t, x, \xi, \gamma)\right| \leq C_{\alpha}(\gamma+|\xi|)^{m-|\alpha|} \log ^{\delta}(1+\gamma+|\xi|)
$$

for all $(t, x, \xi, \gamma)$.

So, take a symbol $a(t, x, \xi, \gamma)$ of order $m \geq 0$, which is log-Zygmund in $t$ and $\log$-Lipschitz in $x$, uniformly with respect to the other variables. Now we smooth $a$ out with respect to time, as done in (3.9). Next lemma, whose proof can be found in [7], provides us estimates on classical symbols associated to $a_{\varepsilon}$ and its time derivatives.

Lemma 3.15. The classical symbols associated to $a_{\varepsilon}$ and its time derivatives satisfy:

$$
\begin{aligned}
\left|\partial_{\xi}^{\alpha} \sigma_{a_{\varepsilon}}\right| & \leq C_{\alpha}(\gamma+|\xi|)^{m-|\alpha|} \\
\left|\partial_{x}^{\beta} \partial_{\xi}^{\alpha} \sigma_{a_{\varepsilon}}\right| & \leq C_{\beta, \alpha}(\gamma+|\xi|)^{m-|\alpha|+|\beta|-1} \log (1+\gamma+|\xi|) \\
\left|\partial_{\xi}^{\alpha} \sigma_{\partial_{t} a_{\varepsilon}}\right| & \leq C_{\alpha}(\gamma+|\xi|)^{m-|\alpha|} \log ^{2}\left(1+\gamma+\frac{1}{\varepsilon}\right) \\
\left|\partial_{x}^{\beta} \partial_{\xi}^{\alpha} \sigma_{\partial_{t} a_{\varepsilon}}\right| & \leq C_{\beta, \alpha}(\gamma+|\xi|)^{m-|\alpha|+|\beta|-1} \log (1+\gamma+|\xi|) \frac{1}{\varepsilon} \\
\left|\partial_{\xi}^{\alpha} \sigma_{\partial_{t}^{2} a_{\varepsilon}}\right| & \leq C_{\alpha}(\gamma+|\xi|)^{m-|\alpha|} \log \left(1+\gamma+\frac{1}{\varepsilon}\right) \frac{1}{\varepsilon} \\
\left|\partial_{x}^{\beta} \partial_{\xi}^{\alpha} \sigma_{\partial_{t}^{2} a_{\varepsilon}}\right| & \leq C_{\beta, \alpha}(\gamma+|\xi|)^{m-|\alpha|+|\beta|-1} \log (1+\gamma+|\xi|) \frac{1}{\varepsilon^{2}}
\end{aligned}
$$

where all the constants which occur here don't depend on the parameter $\gamma$.

Note that first and second inequalities are satisfied also by the symbol $a$ (not smoothed in time).

Now let us quote some basic facts on symbolic calculus, which follow from previous lemma. Before doing this, we recall a definition (see also [17, Def. B.8]).

Definition 3.16. We say that an operator $P$ is of order $m+\delta \log$ if, for every

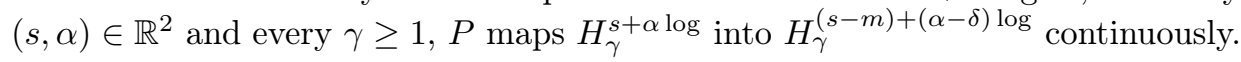

Proposition 3.17. $\quad$ (i) Let a be a symbol of order $m$ which is $L^{\infty}$ in the $x$ variable.

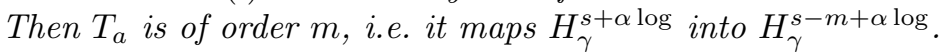

(ii) Let us take two symbols $a, b$ of order $m$ and $m^{\prime}$ respectively. Suppose that $a$, $b$ are $L L$ in the $x$ variable. The composition of the associated operators can be approximated by the symbol associated to the product of these symbols, up to a remainder term:

$$
T_{a} \circ T_{b}=T_{a b}+R .
$$

$R$ has order $m+m^{\prime}-1-\log$ : it maps $H_{\gamma}^{s+\alpha \log }$ into $H_{\gamma}^{s-m-m^{\prime}+1+(\alpha+1) \log }$. 
(iii) Let a be a symbol of order $m$ which is $L L$ in the $x$ variable. The adjoint (over $L^{2}$ ) operator of $T_{a}$ is, up to a remainder operator, $T_{\bar{a}}$. The remainder operator is of order $m-1+\log$, and it maps $H_{\gamma}^{s+\alpha \log }$ into $H_{\gamma}^{s-m+1+(\alpha+1) \log }$.

Let us end this subsection stating a basic positivity estimate. In this situation, paradifferential calculus with parameters comes into play.

Proposition 3.18. Let $a(t, x, \xi, \gamma)$ be a symbol of order $m$, which is $L L$ in the $x$ variable and such that

$$
\operatorname{Re} a(t, x, \xi, \gamma) \geq \lambda_{0}(\gamma+|\xi|)^{m} .
$$

Then, there exists a constant $\lambda_{1}$, depending only on $|a|_{L L}$ and on $\lambda_{0}$ (so not on $\gamma)$, such that, for $\gamma$ large enough, one has

$$
\operatorname{Re}\left(T_{a} u, u\right)_{L^{2}\left(\mathbb{R}_{x}^{N}\right)} \geq \lambda_{1}\|u\|_{H_{\gamma}^{m / 2}}^{2} .
$$

Remark 3.19. If the positive symbol $a$ has low regularity in time and we smooth it out by convolution with respect to this variable, we obtain a family $\left(a_{\varepsilon}\right)_{\varepsilon}$ of positive symbols, with same constant $\lambda_{0}$. Now, all the paradifferential operators associated to these symbols will be positive operators, uniformly in $\varepsilon$ : the constant $\lambda_{1}$ of the previous inequality can be chosen independently of $\varepsilon$.

Then we have the following corollary, which turns out to be fundamental in our energy estimates.

Corollary 3.20. Let a be a positive symbol of order 1 and suppose that a is $L L$ in the $x$ variable.

Then there exists $\gamma \geq 1$, depending only on the symbol a, such that

$$
\left\|T_{a} u\right\|_{L^{2}} \sim\|u\|_{H_{\gamma}^{1}}
$$

for all $u \in H_{\gamma}^{1}\left(\mathbb{R}^{N}\right)$.

\section{Proof of the energy estimates}

Let us now tackle the proof of Theorem 2.2.

The analysis of the higher order terms, which is the most involving part, was already performed in [7]: so, we will give just a sketch of it.

We will give the most of the details, instead, when treating the lower order terms. In doing this, we will follow a different approach than the one used in [9], based on the Bony's paraproduct decomposition (as done in the quoted work [7] for the second order part). 


\subsection{Approximate and total energy}

We first regularize coefficients $a_{i j}$ in the time variable by convolution, as done in (3.9), and define the 0 -th order symbol

$$
\alpha_{\varepsilon}(t, x, \xi):=\left(\gamma^{2}+|\xi|^{2}\right)^{-1 / 2}\left(\gamma^{2}+\sum_{i, j} a_{i j, \varepsilon}(t, x) \xi_{i} \xi_{j}\right)^{1 / 2}
$$

We take $\varepsilon=2^{-\nu}$ (see also [10] and [6]), and (for convenience) we will miss out the $\varepsilon$ in the notation.

We immediately fix a real number $\gamma \geq 1$, which will depend only on $\lambda_{0}$ and on the $\sup _{i, j}\left|a_{i j}\right|_{L L_{x}}$, such that (see Corollary 3.20)

$$
\left\|T_{\alpha^{-1 / 2}} w\right\|_{L^{2}} \geq \frac{\lambda_{0}}{2}\|w\|_{L^{2}} \quad \text { and } \quad\left\|T_{\alpha^{1 / 2}\left(\gamma^{2}+|\xi|^{2}\right)^{1 / 2}} w\right\|_{L^{2}} \geq \frac{\lambda_{0}}{2}\|w\|_{H^{1}}
$$

for all $w \in H^{\infty}$. Recall that the choice of $\gamma$ is equivalent to the choice of the parameter $\mu$ in (3.1) (see Remark 3.6): hence, from now on we will consider paraproducts starting from this $\mu$, according to definition (3.1), even if we will omit it in the notations.

Consider in $(2.1)$ a function $u \in \mathcal{C}^{2}\left(\left[0, T_{0}\right] ; H^{\infty}\right)$. We want to get energy estimates for $u$. We rewrite the equation using paraproduct operators by the coefficients $a_{i j}$ :

$$
\begin{aligned}
\partial_{t}^{2} u & =L u+\sum_{i, j} \partial_{i}\left(a_{i j} \partial_{j} u\right)-\sum_{j=0}^{N} b_{j}(t, x) \partial_{j} u-c(t, x) u \\
& =L u+\sum_{i, j} \partial_{i}\left(\left(T_{a_{i j}}+\widetilde{A}_{i j}\right) \partial_{j} u\right)-\sum_{j=0}^{N}\left(T_{b_{j}}+\widetilde{B}_{j}\right) \partial_{j} u-\left(T_{c}+\widetilde{C}\right) u
\end{aligned}
$$

where $\widetilde{A}_{i j} \partial_{j} u=\left(\left(a_{i j}-T_{a_{i j}}\right) \partial_{j} u\right)$ and analogous for $\widetilde{B}_{j}$ and $\widetilde{C}$. Let us apply the operator $\Delta_{\nu}$ : we get

$$
\begin{aligned}
\partial_{t}^{2} u_{\nu}= & \sum_{i, j} \partial_{i}\left(T_{a_{i j}} \partial_{j} u_{\nu}\right)-\sum_{j} T_{b_{j}} \partial_{j} u_{\nu}-T_{c} u_{\nu}+ \\
& +(L u)_{\nu}+\sum_{i j}\left(\partial_{i}\left(\widetilde{A}_{i j} \partial_{j} u\right)\right)_{\nu}-\sum_{j}\left(\widetilde{B}_{j} \partial_{j} u\right)_{\nu}-(\widetilde{C} u)_{\nu}+ \\
& \quad+\sum_{i, j} \partial_{i}\left(\left[\Delta_{\nu}, T_{a_{i j}}\right] \partial_{j} u\right)-\sum_{j}\left[\Delta_{\nu}, T_{b_{j}}\right] \partial_{j} u-\left[\Delta_{\nu}, T_{c}\right] u
\end{aligned}
$$

where we have set $f_{\nu}=\Delta_{\nu} f$ and $\left[\Delta_{\nu}, T_{g}\right]$ is the commutator operator between $\Delta_{\nu}$ and the paramultiplication by $g$.

Now, we define

$$
\begin{aligned}
v_{\nu}(t, x) & :=T_{\alpha^{-1 / 2}} \partial_{t} u_{\nu}-T_{\partial_{t}\left(\alpha^{-1 / 2}\right)} u_{\nu} \\
w_{\nu}(t, x) & :=T_{\alpha^{1 / 2}\left(\gamma^{2}+|\xi|^{2}\right)^{1 / 2}} u_{\nu} \\
z_{\nu}(t, x) & :=u_{\nu}
\end{aligned}
$$


and the approximate energy associated to $u_{\nu}$ :

$$
e_{\nu}(t):=\left\|v_{\nu}(t)\right\|_{L^{2}}^{2}+\left\|w_{\nu}(t)\right\|_{L^{2}}^{2}+\left\|z_{\nu}(t)\right\|_{L^{2}}^{2} .
$$

Remark 4.1. Note that $\left\|w_{\nu}(t)\right\|_{L^{2}}^{2} \sim\left\|\nabla u_{\nu}\right\|_{L^{2}}^{2} \sim 2^{2 \nu}\left\|u_{\nu}\right\|_{L^{2}}^{2}$, thanks to hypothesis (2.2) and the choice of the frequency $\mu$ from which the paraproduct starts, recall also (4.1).

Now, we fix a $\theta \in] 0,1[$, as required in hypothesis, and we take a $\beta>0$ to be chosen later; we can define the total energy associated to the solution $u$ as

$$
E(t):=\sum_{\nu \geq 0} e^{-2 \beta(\nu+1) t} 2^{-2 \nu \theta} e_{\nu}(t)
$$

It's not difficult to prove (see also inequality (4.8) below) that there exist constants $C_{\theta}$ and $C_{\theta}^{\prime}$, depending only on the fixed $\theta$, for which one has:

$$
\begin{aligned}
& (E(0))^{1 / 2} \leq C_{\theta}\left(\left\|\partial_{t} u(0)\right\|_{H^{-\theta}}+\|u(0)\|_{H^{-\theta+1}}\right) \\
& (E(t))^{1 / 2} \geq C_{\theta}^{\prime}\left(\left\|\partial_{t} u(t)\right\|_{H^{-\theta-\beta^{*} t}}+\|u(t)\|_{H^{-\theta+1-\beta^{*} t}}\right),
\end{aligned}
$$

where we have set $\beta^{*}=\beta(\log 2)^{-1}$.

\subsection{Time derivative of the approximate energy}

Let's find an estimate on the time derivative of the energy. We start analysing each term of (4.3).

4.2.1. $z_{\nu}$ term. For the third term we have:

$$
\frac{d}{d t}\left\|z_{\nu}(t)\right\|_{L^{2}}^{2}=2 \operatorname{Re}\left(u_{\nu}, \partial_{t} u_{\nu}\right)_{L^{2}} .
$$

Now, we have to control the term $\partial_{t} u_{\nu}$ : using positivity of operator $T_{\alpha^{-1 / 2}}$, we get

$$
\begin{aligned}
\left\|\partial_{t} u_{\nu}\right\|_{L^{2}} & \leq C\left\|T_{\alpha^{-1 / 2}} \partial_{t} u_{\nu}\right\|_{L^{2}} \\
& \leq C\left(\left\|v_{\nu}\right\|_{L^{2}}+\left\|T_{\partial_{t}\left(\alpha^{-1 / 2}\right)} u_{\nu}\right\|_{L^{2}}\right) \leq C\left(e_{\nu}\right)^{1 / 2} .
\end{aligned}
$$

So, we find the estimate:

$$
\frac{d}{d t}\left\|z_{\nu}(t)\right\|_{L^{2}}^{2} \leq C e_{\nu}(t)
$$

4.2.2. $v_{\nu}$ term. Straightforward computations show that

$$
\partial_{t} v_{\nu}(t, x)=T_{\alpha^{-1 / 2}} \partial_{t}^{2} u_{\nu}-T_{\partial_{t}^{2}\left(\alpha^{-1 / 2}\right)} u_{\nu} .
$$

Therefore, keeping in mind relation (4.2), we get:

$$
\begin{aligned}
\frac{d}{d t}\left\|v_{\nu}(t)\right\|_{L^{2}}^{2}=-2 \operatorname{Re}\left(v_{\nu}, T_{\partial_{t}^{2}\left(\alpha^{-1 / 2}\right)} u_{\nu}\right)_{L^{2}}+ \\
\quad+2 \sum_{i, j} \operatorname{Re}\left(v_{\nu}, T_{\alpha^{-1 / 2}}\left(\partial_{i}\left(T_{a_{i j}} \partial_{j} u_{\nu}\right)-T_{b_{j}} \partial_{j} u_{\nu}-T_{c} u_{\nu}\right)\right)_{L^{2}}+ \\
\quad+2 \operatorname{Re}\left(v_{\nu}, T_{\alpha^{-1 / 2}}(\widetilde{L} u)_{\nu}\right)_{L^{2}}+2 \operatorname{Re}\left(v_{\nu}, T_{\alpha^{-1 / 2}} \mathcal{X} u\right)_{L^{2}},
\end{aligned}
$$


where we have defined

$$
\begin{aligned}
\widetilde{L} u & :=L u+\sum_{i j} \partial_{i}\left(\widetilde{A}_{i j} \partial_{j} u\right)-\sum_{j} \widetilde{B}_{j} \partial_{j} u-\widetilde{C} u \\
\mathcal{X} u & :=\sum_{i, j} \partial_{i}\left[\Delta_{\nu}, T_{a_{i j}}\right] \partial_{j} u+\sum_{j}\left[\Delta_{\nu}, T_{b_{j}}\right] \partial_{j} u+\left[\Delta_{\nu}, T_{c}\right] u .
\end{aligned}
$$

Obviously, we have

$$
\left|2 \operatorname{Re}\left(v_{\nu}, T_{\alpha^{-1 / 2}}(\widetilde{L} u)_{\nu}\right)_{L^{2}}\right| \leq C\left(e_{\nu}\right)^{1 / 2}\left\|(\widetilde{L} u)_{\nu}\right\|_{L^{2}},
$$

while from Lemma 3.15 and the choice $\varepsilon=2^{-\nu}$ one immediately recovers

$$
\left|2 \operatorname{Re}\left(v_{\nu}, T_{\partial_{t}^{2}\left(\alpha^{-1 / 2}\right)} u_{\nu}\right)_{L^{2}}\right| \leq C(\nu+1) e_{\nu} .
$$

The other terms (paraproducts, commutators) of (4.10) will be treated later.

4.2.3. $w_{\nu}$ term. We now differentiate $w_{\nu}$ in time. A systematic use of symbolic calculus (see [7, Par. 4.2.3] for the details) leads us to

$$
\frac{d}{d t}\left\|w_{\nu}\right\|_{L^{2}}^{2}=2 \operatorname{Re}\left(v_{\nu}, T_{\alpha^{-1 / 2}} T_{\alpha^{2}\left(\gamma^{2}+|\xi|^{2}\right)} u_{\nu}\right)_{L^{2}}+\mathcal{R}_{\nu},
$$

where $\mathcal{R}_{\nu}$ is a suitable remainder term which can be bounded by the localized energy, at the cost of a logarithmic loss:

$$
\left|\mathcal{R}_{\nu}\right| \leq C(\nu+1) e_{\nu}
$$

Remark 4.2. The analysis of the remainder term $\mathcal{R}_{\nu}$ is performed by use of the symbolic calculus, introduced in Section 3. Let us point out that, concerning the regularity in $x$ of the symbols, only the first order derivatives come into play. This fact will be relevant in Section 5, in particular in Lemma 5.1.

Let us now consider the paraproduct terms. First of all, we pair up the second term of (4.10) with the first one of (4.13). As proved in [7], we have

$$
\left|2 \operatorname{Re}\left(v_{\nu}, T_{\alpha^{-1 / 2}}\left(\sum_{i, j} \partial_{i}\left(T_{a_{i j}} \partial_{j} u_{\nu}\right)+T_{\alpha^{2}\left(\gamma^{2}+|\xi|^{2}\right)} u_{\nu}\right)\right)_{L^{2}}\right| \leq C(\nu+1) e_{\nu}
$$

where the constant $C$ depends on the log-Lipschitz norm (with respect to space) of the coefficients $a_{i j}$ and on the fixed parameters $\mu$ and $\gamma$.

For the lower order terms, instead, we just use continuity results for the paraproduct operator (and (4.8) for $j=0$ below):

$$
\begin{aligned}
\left|2 \operatorname{Re}\left(v_{\nu}, T_{\alpha^{-1 / 2}} T_{c} u_{\nu}\right)_{L^{2}}\right| & \leq C e_{\nu} \\
\left|\sum_{j=0}^{N} 2 \operatorname{Re}\left(v_{\nu}, T_{\alpha^{-1 / 2}} T_{b_{j}} \partial_{j} u_{\nu}\right)_{L^{2}}\right| & \leq C e_{\nu},
\end{aligned}
$$

where the constants $C$ depend on $\|c\|_{L^{\infty}\left(\left[0, T_{0}\right] \times \mathbb{R}^{N}\right)}$ and $\sup _{j}\left\|b_{j}\right\|_{L^{\infty}\left(\left[0, T_{0}\right] ; \mathcal{C}^{\omega}\right)}$. 
To sum up, from inequalities (4.9), (4.11), (4.12), from the analysis of the paradifferential operators in (4.15), (4.16) and (4.17), and from the estimate for the remainder term (4.14), we can conclude that

$$
\begin{aligned}
\frac{d}{d t} e_{\nu}(t) \leq C_{1}(\nu+1) e_{\nu}(t)+C_{2}\left(e_{\nu}(t)\right)^{1 / 2}\left\|(\widetilde{L} u)_{\nu}(t)\right\|_{L^{2}}+ \\
+\left|2 \operatorname{Re}\left(v_{\nu}, T_{\alpha^{-1 / 2}} \mathcal{X} u\right)_{L^{2}}\right|
\end{aligned}
$$

\subsection{Commutator terms}

Let us now focus on the commutator terms. We will recall also the most of the details concerning the analysis of the higher order terms, because conditions over $\beta^{*}$ and $T$ in Theorem 2.2 will follow from it.

So, first of all let us consider the quantity

$$
\left|\sum_{i, j} \operatorname{Re}\left(v_{\nu}, T_{\alpha^{-1 / 2}} \partial_{i}\left[\Delta_{\nu}, T_{a_{i j}}\right] \partial_{j} u\right)_{L^{2}}\right|
$$

We start remarking that

$$
\left[\Delta_{\nu}, T_{a_{i j}}\right] w=\left[\Delta_{\nu}, S_{\mu} a_{i j}\right] S_{\mu+2} w+\sum_{k=\mu+3}^{+\infty}\left[\Delta_{\nu}, S_{k-3} a_{i j}\right] \Delta_{k} w
$$

where $\mu$ is fixed, as usual (see Remark 3.6). Consequently,

$$
\begin{aligned}
\partial_{i}\left(\left[\Delta_{\nu}, T_{a_{i j}}\right] \partial_{j} u\right)=\partial_{i}\left(\left[\Delta_{\nu}, S_{\mu} a_{i j}\right]\right. & \left.\partial_{j}\left(S_{\mu+2} u\right)\right)+ \\
& +\partial_{i}\left(\sum_{k=\mu+3}^{+\infty}\left[\Delta_{\nu}, S_{k-3} a_{i j}\right] \partial_{j}\left(\Delta_{k} u\right)\right) .
\end{aligned}
$$

The support of the Fourier transform of $\left[\Delta_{\nu}, S_{\mu} a_{i j}\right] \partial_{j}\left(S_{\mu+2} u\right)$ is contained in $\left\{|\xi| \leq 2^{\mu+4}\right\}$ and moreover $\left[\Delta_{\nu}, S_{\mu} a_{i j}\right] \partial_{j}\left(S_{\mu+2} u\right)$ is identically 0 if $\nu \geq \mu+5$. Then, from Bernstein's inequality and [4, Th. 35$]$ we have

$$
\left\|\partial_{i}\left(\left[\Delta_{\nu}, S_{\mu} a_{i j}\right] \partial_{j}\left(S_{\mu+2} u\right)\right)\right\|_{L^{2}} \leq C_{\mu}\left(\sup _{i, j}\left\|a_{i j}\right\|_{L L_{x}}\right)\left\|S_{\mu+2} u\right\|_{L^{2}}
$$


hence, putting all these facts together, we find

$$
\begin{aligned}
\left|\sum_{\nu=0}^{+\infty} e^{-2 \beta(\nu+1) t} 2^{-2 \nu \theta} \sum_{i j} 2 \operatorname{Re}\left(v_{\nu}, T_{\alpha^{-1 / 2}} \partial_{i}\left(\left[\Delta_{\nu}, S_{\mu} a_{i j}\right] \partial_{j}\left(S_{\mu+2} u\right)\right)\right)_{L^{2}}\right| \leq \quad \text { (4.19) } \\
\leq C_{\mu}\left(\sup _{i, j}\left\|a_{i j}\right\|_{L L_{x}}\right) \sum_{\nu=0}^{\mu+4} e^{-2 \beta(\nu+1) t} 2^{-2 \nu \theta}\left\|v_{\nu}\right\|_{L^{2}}\left(\sum_{h=0}^{\mu+2}\left\|u_{h}\right\|_{L^{2}}\right) \\
\leq C_{\mu}\left(\sup _{i, j}\left\|a_{i j}\right\|_{L L_{x}}\right) e^{\beta(\mu+5) T} 2^{(\mu+4) \theta} \times \\
\quad \times \sum_{\nu=0}^{\mu+4} e^{-\beta(\nu+1) t} 2^{-\nu \theta}\left\|v_{\nu}\right\|_{L^{2}} \sum_{h=0}^{\mu+4} e^{-\beta(h+1) t} 2^{-h \theta}\left\|u_{h}\right\|_{L^{2}} \\
\leq C_{\mu}\left(\sup _{i, j}\left\|a_{i j}\right\|_{L L_{x}}\right) e^{\beta(\mu+5) T} 2^{(\mu+4) \theta} \sum_{\nu=0}^{\mu+4} e^{-2 \beta(\nu+1) t} 2^{-2 \nu \theta} e_{\nu}(t) .
\end{aligned}
$$

Next, let's consider

$$
\partial_{i}\left(\sum_{k=\mu+3}^{+\infty}\left[\Delta_{\nu}, S_{k-3} a_{i j}\right] \partial_{j}\left(\Delta_{k} u\right)\right) .
$$

Looking at the support of the Fourier transform, it is possible to see that

$$
\left[\Delta_{\nu}, S_{k-3} a_{i j}\right] \partial_{j}\left(\Delta_{k} u\right)
$$

is identically 0 if $|k-\nu| \geq 3$. Consequently the sum over $k$ is reduced to at most 5 terms: $\partial_{i}\left(\left[\Delta_{\nu}, S_{\nu-5} a_{i j}\right] \partial_{j}\left(\Delta_{\nu-2} u\right)\right)+\cdots+\partial_{i}\left(\left[\Delta_{\nu}, S_{\nu-1} a_{i j}\right] \partial_{j}\left(\Delta_{\nu+2} u\right)\right)$, each of them having the support of the Fourier transform contained in $\left\{|\xi| \leq 2^{\nu+1}\right\}$. Let's consider one of these terms, e.g. $\partial_{i}\left(\left[\Delta_{\nu}, S_{\nu-3} a_{i j}\right] \partial_{j}\left(\Delta_{\nu} u\right)\right)$, the computation for the other ones being similar. We have, from Bernstein's inequality,

$$
\left\|\partial_{i}\left(\left[\Delta_{\nu}, S_{\nu-3} a_{i j}\right] \partial_{j}\left(\Delta_{\nu} u\right)\right)\right\|_{L^{2}} \leq C 2^{\nu}\left\|\left[\Delta_{\nu}, S_{\nu-3} a_{i j}\right] \partial_{j}\left(\Delta_{\nu} u\right)\right\|_{L^{2}} .
$$

On the other hand, using [4, Th. 35] again, we get

$$
\left\|\left[\Delta_{\nu}, S_{\nu-3} a_{i j}\right] \partial_{j}\left(\Delta_{\nu} u\right)\right\|_{L^{2}} \leq C\left\|\nabla S_{\nu-3} a_{i j}\right\|_{L^{\infty}}\left\|\Delta_{\nu} u\right\|_{L^{2}},
$$

where $C$ does not depend on $\nu$. Consequently, using also (3.7), we deduce

$$
\left\|\partial_{i}\left(\left[\Delta_{\nu}, S_{\nu-3} a_{i j}\right] \partial_{j}\left(\Delta_{\nu} u\right)\right)\right\|_{L^{2}} \leq C 2^{\nu}(\nu+1)\left(\sup _{i, j}\left\|a_{i j}\right\|_{L L_{x}}\right)\left\|\Delta_{\nu} u\right\|_{L^{2}} .
$$

From this last inequality and similar ones for the other terms, we infer

$$
\begin{array}{r}
\left|\sum_{i, j} \operatorname{Re}\left(v_{\nu}, T_{\alpha^{-1 / 2}} \partial_{i}\left(\sum_{k=\mu+3}^{+\infty}\left[\Delta_{\nu}, S_{k-3} a_{i j}\right] \partial_{j}\left(\Delta_{k} u\right)\right)\right)_{L^{2}}\right| \leq \\
\leq C\left(\sup _{i, j}\left\|a_{i j}\right\|_{L L_{x}}\right)(\nu+1) e_{\nu}(t)
\end{array}
$$


and then, keeping in mind also (4.19),

$$
\begin{gathered}
\left|\sum_{\nu=0}^{+\infty} e^{-2 \beta(\nu+1) t} 2^{-2 \nu \theta} \sum_{i j} 2 \operatorname{Re}\left(v_{\nu}, T_{\alpha^{-1 / 2}} \partial_{i}\left[\Delta_{\nu}, T_{a_{i j}}\right] \partial_{j} u\right)_{L^{2}}\right| \leq \\
\leq C \sum_{\nu=0}^{+\infty}(\nu+1) e^{-2 \beta(\nu+1) t} 2^{-2 \nu \theta} e_{\nu}(t),
\end{gathered}
$$

where $C$ depends on $\mu, \sup _{i, j}\left\|a_{i j}\right\|_{L L_{x}}$, on $\theta$ and on the product $\beta T$.

Let us now deal with the commutators coming from the lower order parts. As the analysis is analogous to just performed one, we will only sketch the line of the argument. Moreover, let us consider only the $b_{j}$ 's terms, which are actually worse than the commutator $\left[\Delta_{\nu}, T_{c}\right]$.

First of all, we focus on the case of space derivatives: so, we take $1 \leq j \leq N$. As before, we can write

$$
\left[\Delta_{\nu}, T_{b_{j}}\right] \partial_{j} u=\left[\Delta_{\nu}, S_{\mu} b_{j}\right] \partial_{j} S_{\mu+2} u+\sum_{k=\mu+3}^{+\infty}\left[\Delta_{\nu}, S_{k-3} b_{j}\right] \partial_{j} \Delta_{k} u
$$

From Bernstein's inequality and [4, Th. 35], one gets

$$
\begin{aligned}
\left\|\left[\Delta_{\nu}, S_{\mu} b_{j}\right] \partial_{j} S_{\mu+2} u\right\|_{L^{2}} & \leq C\left\|\nabla S_{\mu} b_{j}\right\|_{L^{\infty}}\left\|S_{\mu+2} u\right\|_{L^{2}} \\
& \leq C_{\mu}\left\|b_{j}\right\|_{\mathcal{C}^{\omega}}\left\|S_{\mu+2} u\right\|_{L^{2}} ;
\end{aligned}
$$

hence, exactly as done in estimate (4.19), we finally have

$$
\begin{aligned}
& \mid \sum_{\nu=0}^{+\infty} e^{-2 \beta(\nu+1) t} 2^{-2 \nu \theta} \sum_{j=1}^{N} 2 \operatorname{Re}\left(v_{\nu},\right.\left.T_{\alpha^{-1 / 2}}\left[\Delta_{\nu}, S_{\mu} b_{j}\right] \partial_{j} S_{\mu+2} u\right)_{L^{2}} \mid \leq \\
& \leq C_{\mu}\left(\sup _{j}\left\|b_{j}\right\|_{\mathcal{C}^{\omega}}\right) e^{\beta(\mu+5) T} 2^{(\mu+4) \theta} E(t) .
\end{aligned}
$$

Moreover, also in this case, the series over $k$ reduces to a finite sum, due to spectral localization of operators $S_{k}$ and $\Delta_{k}$. As before, it's enough to consider only the term for $k=\nu$ : [4, Th. 35] tells us, once again, that

$$
\left\|\left[\Delta_{\nu}, S_{\nu-3} b_{j}\right] \partial_{j} \Delta_{\nu} u\right\|_{L^{2}} \leq C\left\|\nabla S_{\nu-3} b_{j}\right\|_{L^{\infty}}\left\|\Delta_{\nu} u\right\|_{L^{2}} \leq C 2^{\nu}\left\|b_{j}\right\|_{\mathcal{C}^{\omega}}\left\|\Delta_{\nu} u\right\|_{L^{2}},
$$

and therefore we have

$$
\begin{array}{r}
\left|\sum_{\nu=0}^{+\infty} e^{-2 \beta(\nu+1) t} 2^{-2 \nu \theta} \sum_{j=1}^{N} 2 \operatorname{Re}\left(v_{\nu}, T_{\alpha^{-1 / 2}} \sum_{k=\mu+3}^{+\infty}\left[\Delta_{\nu}, S_{k-3} b_{j}\right] \partial_{j} \Delta_{k} u\right)\right| \leq \\
\leq C\left(\sup _{j}\left\|b_{j}\right\|_{\mathcal{C}^{2}}\right) E(t) .
\end{array}
$$

The term involving the time derivative of $u$, instead, requires us a little bit harder work because we can no more shift the derivative on the coefficient $b_{0}$ (as 
done before thanks to [4, Th. 35]). It's not difficult to see that the kernel of the operator

$$
\left[\Delta_{\nu}, S_{\kappa} b_{0}\right]
$$

for all $\kappa \geq 0$, is the function

$$
k(x, y)=2^{\kappa N} \check{\varphi}\left(2^{\kappa}(x-y)\right)\left(S_{\kappa} b_{0}(x)-S_{\kappa} b_{0}(y)\right),
$$

where we have set $\check{\varphi}$ to be the inverse Fourier transform of the localization function $\varphi$ (defined in subsection 3.1). So, by Schur's Lemma the operator norm of the previous commutator over $L^{2}$ is controlled by

$$
\left\|\left[\Delta_{\nu}, S_{\kappa} b_{0}\right]\right\|_{\mathcal{L}\left(L^{2}\right)} \leq C\left\|b_{0}\right\|_{\mathcal{C}^{\omega}} 2^{-\omega \kappa} .
$$

This having been established, again we consider first the term $\left[\Delta_{\nu}, S_{\mu} b_{0}\right] S_{\mu+2} \partial_{t} u$. From the previous estimate we get

$$
\left\|\left[\Delta_{\nu}, S_{\mu} b_{0}\right] S_{\mu+2} \partial_{t} u\right\|_{L^{2}} \leq C\left\|b_{0}\right\|_{\mathcal{C} \omega} 2^{-\omega \mu}\left\|S_{\mu+2} \partial_{t} u\right\|_{L^{2}},
$$

and arguing as for the (4.19) leads us to

$$
\begin{aligned}
&\left|\sum_{\nu=0}^{+\infty} e^{-2 \beta(\nu+1) t} 2^{-2 \nu \theta} 2 \operatorname{Re}\left(v_{\nu}, T_{\alpha^{-1 / 2}}\left[\Delta_{\nu}, S_{\mu} b_{0}\right] S_{\mu+2} \partial_{t} u\right)_{L^{2}}\right| \\
& \leq C\left\|b_{0}\right\|_{\mathcal{C}^{\omega}} e^{\beta(\mu+5) T} 2^{(\mu+4)(\theta-\omega)} E(t) .
\end{aligned}
$$

The same arguments give an analogous estimate for $\left[\Delta_{\nu}, S_{\nu-3} b_{0}\right] \Delta_{\nu} \partial_{t} u$. Therefore the commutator term with first order coefficients can be bounded by

$$
\left|\sum_{\nu=0}^{+\infty} e^{-2 \beta(\nu+1) t} 2^{-2 \nu \theta} \sum_{j=0}^{N} 2 \operatorname{Re}\left(v_{\nu}, T_{\alpha^{-1 / 2}}\left[\Delta_{\nu}, T_{b_{j}}\right] \partial_{j} u\right)_{L^{2}}\right| \leq C E(t),
$$

where $C$ depends also on $\sup _{j}\left\|b_{j}\right\|_{\mathcal{C}^{\omega}}$.

As already said, the commutator term $\left[\Delta_{\nu}, T_{c}\right]$ is actually better, even if $c$ is only $L^{\infty}$, because there are no derivatives acting on $u$. The estimate is finally

$$
\left|\sum_{\nu=0}^{+\infty} e^{-2 \beta(\nu+1) t} 2^{-2 \nu \theta} 2 \operatorname{Re}\left(v_{\nu}, T_{\alpha^{-1 / 2}}\left[\Delta_{\nu}, T_{c}\right] u\right)_{L^{2}}\right| \leq C\|c\|_{L^{\infty}} E(t) .
$$

In the end, putting inequalities (4.20), (4.21) and (4.22) all together, we get

$$
\begin{aligned}
&\left|\sum_{\nu=0}^{+\infty} e^{-2 \beta(\nu+1) t} 2^{-2 \nu \theta} 2 \operatorname{Re}\left(v_{\nu}, T_{\alpha^{-1 / 2}} \mathcal{X} u\right)_{L^{2}}\right| \\
& \leq C_{3} \sum_{\nu=0}^{+\infty}(\nu+1) e^{-2 \beta(\nu+1) t} 2^{-2 \nu \theta} e_{\nu}(t),
\end{aligned}
$$

where $C_{3}$ depends on $\mu$, on the norms of the coefficients on the respective functional spaces, on $\theta$ and on the product $\beta T$. 
4.4. The analysis of $\widetilde{L} u$

From (4.18) and (4.23) we get

$$
\begin{aligned}
\frac{d}{d t} E(t) \leq\left(C_{1}+C_{3}\right. & -2 \beta) \sum_{\nu=0}^{+\infty}(\nu+1) e^{-2 \beta(\nu+1) t} 2^{-2 \nu \theta} e_{\nu}(t)+ \\
& +C_{2} \sum_{\nu=0}^{+\infty} e^{-2 \beta(\nu+1) t} 2^{-2 \nu \theta}\left(e_{\nu}(t)\right)^{1 / 2}\left\|(\widetilde{L} u(t))_{\nu}\right\|_{L^{2}} .
\end{aligned}
$$

Let us now consider each term of $\widetilde{L} u$ separately. From Hölder inequality we immediately gather

$$
\sum_{\nu=0}^{+\infty} e^{-2 \beta(\nu+1) t} 2^{-2 \nu \theta}\left(e_{\nu}(t)\right)^{1 / 2}\left\|(L u)_{\nu}\right\|_{L^{2}} \leq C(E(t))^{1 / 2}\|L u(t)\|_{H^{-\theta-\beta^{*} t}} .
$$

Applying Hölder inequality for series again implies (see also [7, Sect. 4.4] for the details)

$$
\begin{aligned}
& \sum_{\nu=0}^{+\infty} e^{-2 \beta(\nu+1) t} 2^{-2 \nu \theta}\left(e_{\nu}(t)\right)^{1 / 2}\left\|\left(\sum_{i, j} \partial_{i}\left(\widetilde{A}_{i j} \partial_{j} u\right)\right)\right\|_{\nu} \leq \\
& \quad \leq\left(\sum_{\nu=0}^{+\infty}(\nu+1) e^{-2 \beta(\nu+1) t} 2^{-2 \nu \theta} e_{\nu}(t)\right)^{1 / 2}\left\|\sum_{i, j} \partial_{i}\left(\widetilde{A}_{i j} \partial_{j} u\right)\right\|_{H^{-\theta-\beta^{*} t-\frac{1}{2} \log }},
\end{aligned}
$$

and from [11, Prop. 3.4] we have

$$
\left\|\sum_{i, j} \partial_{i}\left(\widetilde{A}_{i j} \partial_{j} u\right)\right\|_{H^{-s-\frac{1}{2} \log }} \leq C\left(\sup _{i, j}\left\|a_{i j}\right\|_{L L_{x}}\right)\|u\|_{H^{1-s+\frac{1}{2} \log }},
$$

with $C$ uniformly bounded for $s$ in a compact set of $] 0,1[$. Consequently,

$$
\begin{aligned}
\left\|\sum_{i, j} \partial_{i}\left(\widetilde{A}_{i j} \partial_{j} u\right)\right\|_{H^{-\theta-\beta^{*} t-\frac{1}{2} \log }} & \leq C\left(\sup _{i, j}\left\|a_{i j}\right\|_{L L_{x}}\right)\|u\|_{H^{1-\theta-\beta^{*} t+\frac{1}{2} \log }} \\
& \leq C\left(\sum_{\nu=0}^{+\infty}(\nu+1) e^{-2 \beta(\nu+1) t} 2^{-2 \nu \theta} e_{\nu}(t)\right)^{1 / 2}
\end{aligned}
$$

and finally

$$
\begin{aligned}
\sum_{\nu=0}^{+\infty} e^{-2 \beta(\nu+1) t} 2^{-2 \nu \theta}\left(e_{\nu}(t)\right)^{1 / 2} \| & \left(\sum_{i, j} \partial_{i}\left(\widetilde{A}_{i j} \partial_{j} u\right)\right) \|_{\nu} \leq \\
& \leq C_{4} \sum_{\nu=0}^{+\infty}(\nu+1) e^{-2 \beta(\nu+1) t} 2^{-2 \nu \theta} e_{\nu}(t)
\end{aligned}
$$

with $C_{4}$ uniformly bounded for $\beta^{*} t+\theta$ in a compact set of $] 0,1[$. 
For the lower order terms, let us state a lemma, which is the analogous of [11, Prop. 3.4].

Lemma 4.3. Given a function $f$, we define the remainder operator

$$
\widetilde{F} u:=f u-T_{f} u \text {. }
$$

(i) If $f \in \mathcal{C}^{\omega}\left(\mathbb{R}^{N}\right)$ for some $\omega>0$ (with $f \in \mathcal{C}_{*}^{\omega}:=B_{\infty, \infty}^{\omega}$ if $\omega \in \mathbb{N}$ ), then $\widetilde{F}$ maps $H^{-s}\left(\mathbb{R}^{N}\right)$ into $H^{\omega-s}\left(\mathbb{R}^{N}\right)$ continuously for all $\left.s \in\right] 0, \omega[$.

(ii) If $f$ is only $L^{\infty}\left(\mathbb{R}^{N}\right)$, then $\widetilde{F}$ maps continuously $H^{s}\left(\mathbb{R}^{N}\right)$ into $L^{2}\left(\mathbb{R}^{N}\right)$ for all $s \geq 0$.

Moreover, the operator norms of $\widetilde{F}$ depend on the norm of $f$ in the respective functional space, and they are uniformly bounded for s varying in a compact subset of $] 0, \omega[$ and $[0,+\infty[$ respectively.

Proof. (i) We can write

$$
\widetilde{F} u=\sum_{\kappa \geq \mu-3} S_{\kappa+2} u \Delta_{\kappa} f=\sum_{\kappa} T_{\kappa} .
$$

As each term $T_{\kappa}$ is spectrally supported in a ball of size $2^{\kappa}$ and $\omega-s>0$, by [1, Lemma 2.84] it's enough to estimate its $L^{2}$ norm. By [1, Prop. 2.79] and dyadic characterization of Hölder spaces (see e.g. [16]), we have

$\left\|S_{\kappa+2} u \Delta_{\kappa} f\right\|_{L^{2}} \leq\left\|S_{\kappa+2} u\right\|_{L^{2}}\left\|\Delta_{\kappa} f\right\|_{L^{\infty}} \leq C\|f\|_{\mathcal{C}^{\omega}}\|u\|_{H^{-s}} 2^{(s-\omega) \kappa} \lambda_{\kappa}$,

for a sequence $\left(\lambda_{\kappa}\right)_{\kappa} \in \ell^{2}$. Therefore we immediately get

$$
\|\widetilde{F} u\|_{H^{\omega-s}} \leq C\|f\|_{\mathcal{C}^{\omega}}\|u\|_{H^{-s}} .
$$

(ii) Thanks to continuity properties of the paraproduct operator, we have

$$
\left\|T_{f} u\right\|_{H^{s}} \leq C\|f\|_{L^{\infty}}\|u\|_{H^{s}}
$$

for any $s \in \mathbb{R}$ : in particular, if $s \geq 0$ the $L^{2}$ norm can be bounded by the previous quantity. Moreover, if $s \geq 0$, obviously

$$
\|f u\|_{L^{2}} \leq\|f\|_{L^{\infty}}\|u\|_{L^{2}} \leq\|f\|_{L^{\infty}}\|u\|_{H^{s}} .
$$

The lemma is completely proved.

Let us come back to our estimates. As done before, we find

$$
\begin{aligned}
\sum_{\nu=0}^{+\infty} e^{-2 \beta(\nu+1) t} 2^{-2 \nu \theta}\left|\sum_{j=0}^{N} 2 \operatorname{Re}\left(v_{\nu}, T_{\alpha^{-1 / 2}}\left(\widetilde{B}_{j} \partial_{j} u\right)_{\nu}\right)_{L^{2}}\right| \leq \\
\leq\left(\sum_{\nu=0}^{+\infty} e^{-2 \beta(\nu+1) t} 2^{-2 \nu \theta} 2^{-2 \nu \omega} e_{\nu}\right)^{1 / 2}\left\|\sum_{j=0}^{N} \widetilde{B}_{j} \partial_{j} u\right\|_{H^{\omega-\theta-\beta^{*} t}} .
\end{aligned}
$$


At this point, Lemma 4.3 immediately tells us

$$
\sum_{\nu=0}^{+\infty} e^{-2 \beta(\nu+1) t} 2^{-2 \nu \theta}\left|\sum_{j=0}^{N} 2 \operatorname{Re}\left(v_{\nu}, T_{\alpha^{-1 / 2}}\left(\widetilde{B}_{j} \partial_{j} u\right)_{\nu}\right)_{L^{2}}\right| \leq C_{5} E(t),
$$

where the constant $C_{5}$ depends also on $\sup _{j}\left\|b_{j}\right\|_{\mathcal{C}^{\omega}}$.

For $\widetilde{C} u$ we argue as before: by Hölder inequality we have

$$
\sum_{\nu=0}^{+\infty} e^{-2 \beta(\nu+1) t} 2^{-2 \nu \theta}\left|\sum_{j=0}^{N} 2 \operatorname{Re}\left(v_{\nu}, T_{\alpha^{-1 / 2}}(\widetilde{C} u)_{\nu}\right)_{L^{2}}\right| \leq C E^{1 / 2}\|\widetilde{C} u\|_{H^{-\theta-\beta^{*} t}},
$$

and so, applying Lemma 4.3 again,

$$
\sum_{\nu=0}^{+\infty} e^{-2 \beta(\nu+1) t} 2^{-2 \nu \theta}\left|\sum_{j=0}^{N} 2 \operatorname{Re}\left(v_{\nu}, T_{\alpha^{-1 / 2}}(\widetilde{C} u)_{\nu}\right)_{L^{2}}\right| \leq C_{6} E(t)
$$

for $C_{6}$ depending on $\|c\|_{L^{\infty}\left(\left[0, T_{0}\right] \times \mathbb{R}^{N}\right)}$.

\subsection{Final estimates}

Now we take $\beta>0\left(\right.$ recall that $\left.\beta^{*}=\beta(\log 2)^{-1}\right)$ and $\left.\left.T \in\right] 0, T_{0}\right]$ such that

$$
\beta^{*} T=\delta<\min \{1, \omega\}-\theta ;
$$

in particular, we have $0<\theta \leq \theta+\beta^{*} t \leq \theta+\delta<1$.

Therefore, putting inequalities (4.25), (4.27), (4.28) and (4.29) into (4.24) we finally find

$$
\begin{aligned}
& \frac{d}{d t} E(t) \leq(K-2 \beta) \sum_{\nu=0}^{+\infty}(\nu+1) e^{-2 \beta(\nu+1) t} 2^{-2 \nu \theta} e_{\nu}(t)+ \\
& \quad+C_{2} \sum_{\nu=0}^{+\infty} e^{-2 \beta(\nu+1) t} 2^{-2 \nu \theta}\left(e_{\nu}(t)\right)^{1 / 2}\left\|(L u(t))_{\nu}\right\|_{L^{2}},
\end{aligned}
$$

where we have set $K=C_{1}+C_{4} C_{2}+C_{3}+C_{5} C_{2}+C_{6} C_{2}$.

Now we fix $\beta$ large enough, such that $K-2 \beta \leq 0$ : this corresponds to take $T>0$ small enough in (4.30). Hence, we finally get the estimate

$$
\frac{d}{d t} E(t) \leq C_{2}(E(t))^{1 / 2}\|L u(t)\|_{H^{-\theta-\beta^{*} t}}
$$

by Gronwall's Lemma and relations (4.5) and (4.6) we infer estimate (2.6).

Remark 4.4. Let us point out that condition (4.30) gives a bound on the lifespan $T$ of a solution to the Cauchy problem for (2.1). It depends on $\theta \in] 0,1[$ and on $\beta^{*}>0$, hence on constants $C_{1} \ldots C_{6}$. Going after the guideline of the proof, one can see that, in the end, $T$ depends only on the index $\theta$, on the parameter $\mu$ defined by conditions (4.1), on constants $\lambda_{0}$ and $\Lambda_{0}$ defined by (2.2), and on the quantities $\sup _{i, j}\left|a_{i j}\right|_{L Z_{t}}, \sup _{i, j}\left|a_{i j}\right|_{L L_{x}}, \sup _{j}\left\|b_{j}\right\|_{L^{\infty}\left(\left[0, T_{0}\right] ; \mathcal{C}^{\omega}\right)}$ and $\|c\|_{L^{\infty}\left(\left[0, T_{0}\right] \times \mathbb{R}^{N}\right)}$. 


\section{The well-posedness in $H^{\infty}$}

In this section we want to sketch how recovering the thesis of Theorem 2.1 from Theorem 2.2. We will follow the outline of the proof in [10] for isotropic logLipschitz coefficients.

So, let us impose more regularity with respect to $x$ on the coefficients. A preliminary lemma is in order.

Lemma 5.1. Consider the operator $L$ defined by (2.1), with coefficients $a_{i j}$ fulfilling conditions (2.2) and (2.3).

Assume that $a_{i j} \in L^{\infty}\left(\left[0, T_{0}\right] ; \mathcal{C}_{b}^{2}\left(\mathbb{R}^{N}\right)\right)$, while $b_{j}$ and $c \in L^{\infty}\left(\left[0, T_{0}\right] ; \mathcal{C}_{b}^{1}\left(\mathbb{R}^{N}\right)\right)$.

Then there exist a $\beta^{*}>0$, a time $T>0$ and a constant $C>0$ such that estimate (2.6) holds true also for $\theta=0$.

Proof. First of all, we note that, thanks to the additional regularity on the principal part of $L$, it makes sense to consider the problem in $H^{1} \times L^{2}$.

Now, it's enough to retake the same steps of the previous proof.

The fundamental fact is that, taking into account the additional regularity of the coefficients of $L$, the logarithmic loss disappears whenever space derivatives of the coefficients are involved. Nevertheless, this is not the case when the time derivative $\partial_{t}$ acts on the symbol $\alpha$ : the loss in the energy estimates comes from these terms.

Taking into account Remark 4.4, note that the lifespan $T$ depends only on the constants $\lambda_{0}$ and $\Lambda_{0}$ (recall (2.2)) and on the norms of the coefficients of $L$ in their respective functional spaces.

Let us now analyse what happens to the space derivatives of $u$.

Lemma 5.2. Consider the operator $L$ defined by (2.1), with coefficients $a_{i j}$ fulfilling conditions (2.2) and (2.3).

Assume that coefficients $a_{i j}, b_{j}$ and $c$ all belong to the space $L^{\infty}\left(\left[0, T_{0}\right] ; \mathcal{C}_{b}^{\infty}\left(\mathbb{R}^{N}\right)\right)$.

Then, for all $m \geq 0$, there exists a constant $C_{m}>0$ such that, for all, $u \in \mathcal{C}^{2}\left([0, T] ; H^{\infty}\left(\mathbb{R}^{N}\right)\right)$, one has the following estimate:

$$
\begin{aligned}
& \sup _{0 \leq t \leq T}\left(\|u(t, \cdot)\|_{H^{m+1-\beta^{*} t}}+\left\|\partial_{t} u(t, \cdot)\right\|_{H^{m-\beta^{*} t}}\right) \leq \\
& \quad \leq C\left(1+e^{C_{m} T}\right)\left(\|u(0, \cdot)\|_{H^{m+1}}+\left\|\partial_{t} u(0, \cdot)\right\|_{H^{m}}+\int_{0}^{T}\|L u(t, \cdot)\|_{H^{m-\beta^{*}} t} d t\right) .
\end{aligned}
$$

Here, the "loss index" $\beta^{*}$, the time $T$ and the constant $C$ are the same of those in Lemma 5.1.

Proof. The proof is analogous to that of [10, Th. 2.3]. Hence, let us just give a sketch of it.

For notation convenience, we define

$$
X_{m}(t):=\int_{0}^{t}\left(\|u(\tau)\|_{H^{m+1-\beta^{*} \tau}}+\left\|\partial_{t} u(\tau)\right\|_{H^{m-\beta^{*} \tau}}\right) d \tau
$$


and the energy of the data

$$
\mathcal{U}(t):=\|u(0)\|_{H^{m+1}}+\left\|\partial_{t} u(0)\right\|_{H^{m}}+\int_{0}^{t}\|L u(\tau)\|_{H^{m-\beta^{*} \tau}} d \tau .
$$

We set moreover $\Lambda\left(D_{x}\right):=\left(1+\left|D_{x}\right|^{2}\right)^{1 / 2}$.

From Lemma 5.1, for any $m \geq 0$ we immediately get

$$
X_{m}^{\prime}(t) \leq C_{0}\left(\mathcal{U}(t)+\int_{0}^{t}\left\|\left[L, \Lambda^{m}\left(D_{x}\right)\right] u(\tau)\right\|_{H^{-\beta^{*} \tau}}\right) d \tau .
$$

Now, differentiating equation (2.1) with respect to $x$, for any multi-index $\eta$ such that $|\eta|=m$ we find

$$
\begin{aligned}
L\left(D_{x}^{\eta} u\right)-D_{x}^{\eta}(L u)= & \sum_{i, j=1}^{N} \sum_{1 \leq|\varrho| \leq m} \partial_{i}\left(D_{x}^{\varrho} a_{i j} \partial_{j} D_{x}^{\eta-\varrho} u\right)+ \\
& -\sum_{j=0}^{N} \sum_{1 \leq|\varrho| \leq m} D_{x}^{\varrho} b_{j} \partial_{j} D_{x}^{\eta-\varrho} u-\sum_{1 \leq|\varrho| \leq m} D_{x}^{\varrho} c D_{x}^{\eta-\varrho} u
\end{aligned}
$$

and we apply Lemma 5.1. Second, third and fourth terms on the right-hand side of the previous equality are obviously of lower order. Hence, we finally get

$$
X_{m}^{\prime}(t) \leq C_{0} \mathcal{U}(t)+C_{m} X_{m}(t),
$$

for a constant $C_{m}$ depending only on the $L^{\infty}$ norms of the coefficients of $L$ and their space derivatives.

Therefore, by Gronwall's inequality we infer

$$
X_{m}(t) \leq \frac{C_{0}}{C_{m}} e^{C_{m} t} \mathcal{U}(t)
$$

which immediately implies estimate (5.1).

Now, Theorem 2.1 easily follows from Lemma 5.2.

Let us just point out that the global in time issue can be obtained remarking that the constants which come into play in the previous proof depend only on the operator $L$ (or better, on its coefficients), and not on the initial data.

\section{References}

[1] H. Bahouri, J.-Y. Chemin and R. Danchin: "Fourier Analysis and Nonlinear Partial Differential Equations", Grundlehren der Mathematischen Wissenschaften (Fundamental Principles of Mathematical Sciences), 343, Springer, Heidelberg, 2011.

[2] J.-M. Bony: Calcul symbolique et propagation des singularités pour les équations aux dérivées partielles non linéaires, Ann. Sci. École Norm. Sup. (4), 14 (1981), 209-246.

[3] M. Cicognani and F. Colombini: Modulus of continuity of the coefficients and loss of derivatives in the strictly hyperbolic Cauchy problem, J. Differential Equations, 221 (2006), 143-157. 
[4] R. Coifman and Y. Meyer: Au delà des opérateurs pseudo-différentiels, Astérisque, 57 (1978), Société Mathématique de France, Paris.

[5] F. Colombini, E. De Giorgi and S. Spagnolo: Sur les équations hyperboliques avec des coefficients qui ne dépendent que du temps, Ann. Scuola Norm. Sup. Pisa Cl. Scienze (4), 6 (1979), 511-559.

[6] F. Colombini and D. Del Santo: A note on hyperbolic operators with log-Zygmund coefficients, J. Math. Sci. Univ. Tokyo, 16 (2009), 95-111.

[7] F. Colombini, D. Del Santo, F. Fanelli and G. Métivier: Time-dependent loss of derivatives for hyperbolic operators with non-regular coefficients, submitted (2012).

[8] F. Colombini, D. Del Santo, F. Fanelli and G. Métivier: A well-posedness result for hyperbolic operators with Zygmund coefficients, J. Math. Pures Appl. (to appear, 2012).

[9] F. Colombini and F. Fanelli: A note on non-homogeneous hyperbolic operators with low regularity coefficients, Rend. Istit. Mat. Univ. Trieste, vol. 42 suppl. (2010).

[10] F. Colombini and N. Lerner: Hyperbolic operators with non-Lipschitz coefficients, Duke Math. J., 77 (1995), 657-698.

[11] F. Colombini and G. Métivier, The Cauchy problem for wave equations with nonLipschitz coefficients; application to continuation of solutions of some nonlinear wave equations, Ann. Sci. École Norm. Sup. (4) 41 (2008), 177-220.

[12] D. Del Santo: The Cauchy problem for a hyperbolic operator with Log-Zygmund coefficients, Further Progress in Analysis, World Sci. Publ., Hackensack, NJ (2009), 425-433.

[13] L. Hörmander: "Linear partial differential operators", Springer-Verlag, Berlin, 1963.

[14] A. E. Hurd, D. H. Sattinger: Questions of existence and uniqueness for hyperbolic equations with discontinuous coefficients, Trans. Amer. Math. Soc., 132, 159-174 (1968).

[15] G. Métivier, Interactions de deux chocs pour un système de deux lois de conservation, en dimension deux d'espace, Trans. Amer. Math. Soc., 296 (1986), 431-479.

[16] G. Métivier, Para-differential calculus and applications to the Cauchy problem for nonlinear systems, Centro di Ricerca Matematica "Ennio De Giorgi" (CRM) Series, 5, Edizioni della Normale, Pisa (2008).

[17] G. Métivier and K. Zumbrun, Large viscous boundary layers for noncharacteristic nonlinear hyperbolic problems, Mem. Amer. Math. Soc., 175 (2005).

[18] S. Mizohata: "The Theory of Partial Differential Equations", Cambridge University Press, Cambridge, 1973.

[19] S. Tarama: Energy estimate for wave equations with coefficients in some Besov type class, Electron J. Differential Equations (2007), Paper No. 85 (electronic).

\section{Acknowledgment}

The third author was partially supported by Grant MTM2011-29306-C02-00, MICINN, Spain, ERC Advanced Grant FP7-246775 NUMERIWAVES, ESF Research Networking Programme OPTPDE and Grant PI2010-04 of the Basque Government. 
Ferruccio Colombini

Dipartimento di Matematica

Università di Pisa

Largo Bruno Pontecorvo, 5

56127 Pisa

Italy

e-mail: colombini@dm.unipi.it

Daniele Del Santo

Dipartimento di Matematica e Geoscienze

Università degli Studi di Trieste

Via Valerio, 12/1

34127 Trieste

Italy

e-mail: delsanto@units.it

Francesco Fanelli

BCAM - Basque Center for Applied Mathematics

Alameda de Mazarredo, 14

E48009 Bilbao

Basque Country, Spain

e-mail: ffanelli@bcamath.org

Guy Métivier

Institut de Mathématiques de Bordeaux, UMR 5251

Univeristé de Bordeaux 1

351, Cours de la Libération

F33405 Talence

France

e-mail: guy.metivier@math.u-bordeaux1.fr 